\title{
Novel Use of deutetrabenazine for refractory chorea hyperglycemia basal ganglia syndrome
}

\author{
M. Faisal Khan ${ }^{1,2 *}$, Angela Welty ${ }^{2}$ and Ahmed Naffi ${ }^{2}$ \\ ${ }^{1}$ Sam Houston State University - College of Osteopathic Medicine, Huntsville, TX, USA \\ ${ }^{2}$ Sugar Land Neurology and Sleep, MD. PA.,1111 HWY 6, STE 275, Sugar Land, TX, USA
}

\section{Case report}

We present to our knowledge the first use of deutetrabenazine in a video case study of Chorea Hyperglycemia Basal Ganglia Syndrome (CHBGS). An 85-year-old Caucasian female presented to her primary care physician with acute/subacute-onset of persistent, irregular, right lower-extremity jerking, relieved only by sleep (Video 1). Her quality of life was significantly affected due to her inability to ambulate, prompting hospitalization. Past medical history was significant for diabetes, hypertension, pulmonary embolism, and recent hospitalization three weeks prior for nonketotic hyperglycemia of $700 \mathrm{mg} / \mathrm{dl}$. No prior neuroleptic exposure was reported. Laboratory work-up showed blood glucose of $200 \mathrm{mg} / \mathrm{dl}$ with HgbA1c of 6.9. Examination revealed no cortical or cranial nerve deficits; movements were choreiformic, without associated ballism, athetosis, or myoclonus. She was unable to perform right heel-to-shin testing, with remaining cerebellar functions, motor strength, and reflexes intact. Broad differential was considered, in light of vascular risk factors, metabolic derangements, and age-of-onset. MRI without contrast showed T1-hyperintensity in the left lentiform nucleus without mass effect; findings consistent with CHBGS. Correction of hyperglycemia did not resolve symptoms. Trials of clonazepam, diazepam, haloperidol, and physical therapy were ineffective. She was discharged to a skilled nursing facility as she was unable to ambulate independently. A trial of deutetrabenazine was initiated on neurology outpatient visit, with marked improvement by the second dose. With continued use of deutetrabenazine (obtained through a compassionate-care program), choreiform movements were essentially resolved and she was able to ambulate with minimal

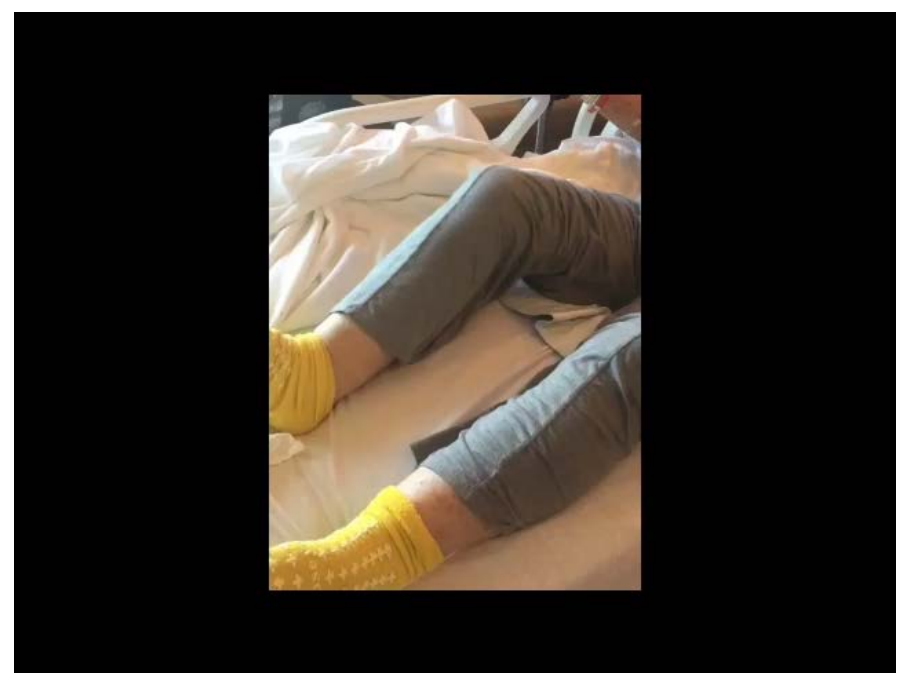

assistance (Video). Attempts to wean off deutetrabenazine resulted in a return of symptoms, which promptly remitted upon reinstitution. No side-effects were reported at regular outpatient follow-up for two years, with resolution of T1-hyperintensity on repeat MRI.

Acute sporadic hemichorea has a broad differential including vascular (acute subthalamic stroke), paraneoplastic (IgLON5, CRMP5), immune (antiphospholipid antibody syndrome), metabolic (nonketotic hyperglycemia) and medications (withdrawal-emergent chorea) [1]. CHBG is a rare complication of nonketotic hyperglycemia. Pathogenesis remains elusive, with several hypotheses proposed, including hypoperfusion and activation of anaerobic metabolism leading to GABA depletion, causing disinhibition of the thalamocortical pathway and motor-cortical hyperexcitability. Histology shows selective neuronal loss, gliosis, and reactive astrocytes without hemorrhage or infarct within the striatum [2]. MRI reveals T1-weighted hyperintensity of the lentiform nucleus with characteristic involvement of the putamen (Figure 1). Prognosis is excellent and most often resolves with achieving normoglycemia, though rare refractory cases have been reported in the literature $[1,3]$. Pharmacological options include neuroleptics (haloperidol/olanzapine), GABA-enhancing antiepileptics (benzodiazepines or topirimate), and central dopamine-depleting agents, specifically vesicular monoamine transporter2 (VMAT2) inhibitors (tetrabenazine) [4].

VMAT2 is located on synaptic vesicles of dopamine, norepinephrine, serotonin, and histamine neurons exclusively within the central nervous system. Tetrabenazine, introduced in 2008, was the first of this class approved for Huntington's chorea. Unfortunately, frequent dosing, along with a poor side-effect profile, resulted in noncompliance [5]. Deutetrabenazine, FDA-approved in 2017, has a better pharmacokinetic and side-effect profile through reduced hepatic CYP2D metabolism due to deuterated hydrogens, giving similar therapeutic effects at decreased dosages [5]. We suggest that the novel use of deutetrabenazine can be applied for patients with refractory CHBG, with excellent clinical outcomes and tolerability.

\section{Author contributions}

M. Faisal Khan: study concept and design, acquisition, analysis, and interpretation of data, draft of manuscript.

${ }^{*}$ Correspondence to: M. Faisal Khan, MD, Clinical Assistant Professor of Neurology, Sam Houston State University-College of Osteopathic Medicine, Huntsville, TX, USA, Tel: 713-234-7132; E-mail: sugarlandneurologist@gmail.com

Received: June 10, 2020; Accepted: June 17, 2020; Published: June 19, 2020 


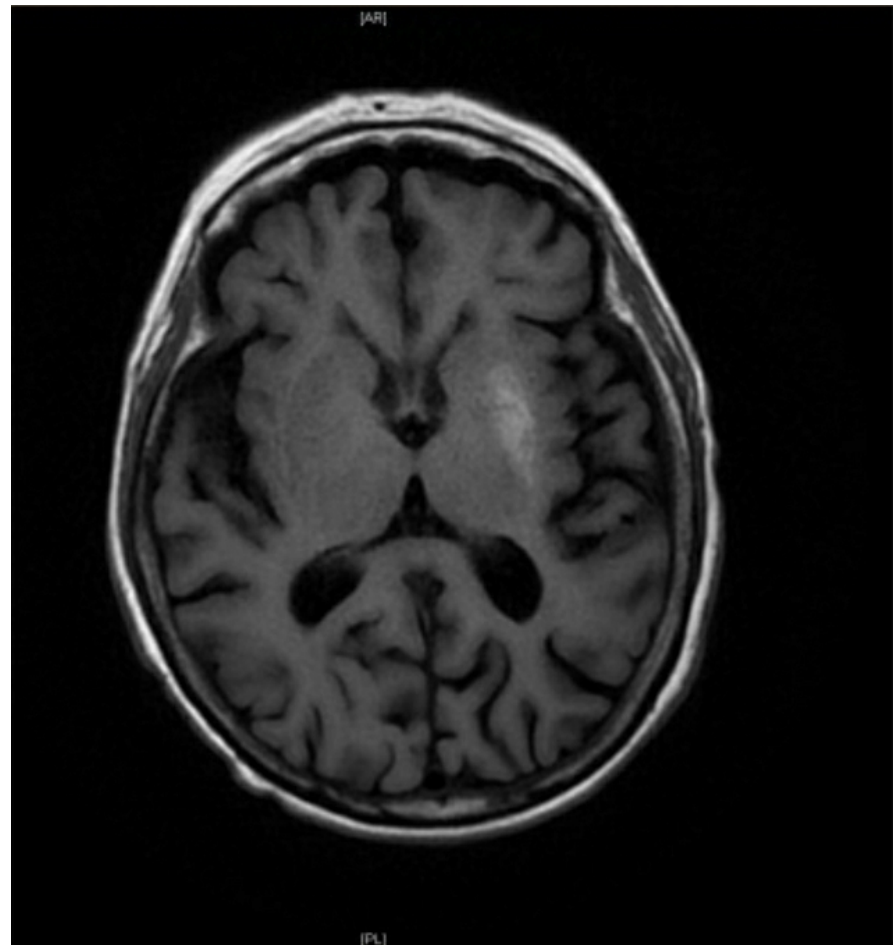

Figure 1. Contralateral, asymmetric, lentiform nucleus T1-hyperintensity was observed, with no associated mass effect or hemorrhage. This radiologic finding is the hallmark of CHBGS

Angela Welty: study concept and design, acquisition, analysis, and interpretation of data, draft of manuscript.
Ahmed Naffi: study concept and design, acquisition, analysis, interpretation of data, draft of manuscript, critical revision of manuscript for intellectual content.

\section{Ethical compliance}

Written informed consent was obtained per local and national guidelines for video-recording the patient and publishing her video and history. We confirm that we have read and are in full compliance with this journal's requirements for ethical publication.

\section{Funding sources and conflict of interest}

All Authors report no sources of funding, and report no conflicts of interest of any kind.

\section{Financial disclosures for the previous 12 months}

All authors have no financial disclosures to disclose for the past 12 months or prior, relevant to the manuscript.

\section{References}

1. Panzer J, Dalmau J (2011) Movement disorders in paraneoplastic and autoimmune disease. Curr Opin Neurol 24: 346-353.

2. Sperling M, Bhowansing R (2018) Case Reports in Medicine. Case Rep Med 2018: 1-5

3. Ahlskog JE, Nishino H, Evidente VG, Tulloch JW, Forbes GS, et al. (2001) Persistent chorea triggered by hyperglycemic crisis in diabetics. Movement disorders 16: 890898

4. McCullen MK, Miller J, Jabbour S, Furlong K, Shirodakar M, et al. (2010) Chorea in the setting of hyperglycemia: A case report and review of the literature Pract Neurol pp: 16-19.

5. Dean M, Sung VW (2018) Review of deutetrabenazine: a novel treatment for chorea associated with Huntington's disease. Drug Des Devel Ther 12: 313-319

Copyright: $\odot 2020$ Khan MF. This is an open-access article distributed under the terms of the Creative Commons Attribution License, which permits unrestricted use, distribution, and reproduction in any medium, provided the original author and source are credited. 